\title{
SUMMABILITY VIEWED AS INTEGRATION ${ }^{1}$
}

\author{
BY GEORGE BRAUER
}

Communicated by R. C. Buck, December 15, 1967

1. Let $s=\left\{s_{n}\right\}$ denote an infinite sequence of complex numbers and let $A=\left(a_{n k}\right)$ be a summation matrix. If the $A$-transform of $s$, $\left\{t_{n}\right\}=\left\{\sum_{k=0}^{\infty} a_{n k} s_{k}\right\}$ is a bounded sequence, it may be regarded as a bounded continuous function $t(n)$ on the discrete space of natural numbers $N$, and thus it has a continuous extension $\tilde{t}$ to $\beta N$, the StoneCech compactification of $N$, cf. [2, pp. 82-95]. Let $\gamma_{0}$ be a fixed point of $\beta N-N$; we define

$$
\int_{N} s d A=\tilde{t}\left(\gamma_{0}\right)
$$

to obtain a finitely additive integration process on $N$. In particular $\int_{N} s d A=\sigma$ whenever the matrix $A$ evaluates $s$ to $\sigma$.

Analogously an integration process on $N$ can be created from summation methods arising from sequence to function transformations. For example if $a$ is the Abel method, we choose a point $\rho_{0}$ in $\beta I-I$, where $I$ denotes the interval $[0,1)$, and define, for all sequences $\left\{s_{n}\right\}$ such that $S(x)=(1-x) \sum_{n=0}^{\infty} s_{n} x^{n}$ converges for $|x|<1$ and is bounded on $I$,

$$
\int_{N} s d Q=\tilde{S}\left(\rho_{0}\right)
$$

where $\tilde{S}$ is the extension of $S$ to $I$. The Abel method gives rise to a translation invariant integration on $N$.

In this note we shall study the function and in particular the Fourier analysis of the integration described. Each summation method will be identified with the measure or integration on $N$ which it defines. All measures will be assumed to be regular summation methods on the set of null sequences; if the measure is representable by a matrix $\left(a_{n k}\right)$ this means

$$
\lim _{n \rightarrow \infty} a_{n, k}=0, \quad \operatorname{lub} \sum_{k=0}^{\infty}\left|a_{n k}\right|<\infty .
$$

REMARK. The only countably additive summation methods $\phi$ are those of the form

1 This research was supported by NSFG 7686. 


$$
\int_{N} s d \phi=\sum_{n=0}^{\infty} a_{n} s_{n}
$$

where $\left\{a_{n}\right\}$ is a sequence of numbers such that the right-hand side of (2) exists. If $\phi$ is nonatomic, then it is purely finitely additive [3, p. 163].

For each summation method $\phi$ we can define the space $\mathscr{L}^{p}(\phi)$ of sequences such that $\int_{N}\left|s_{n}\right|{ }^{p} d \phi$ exists (we identify two sequences $s$ and $t$ such that $\left.\int_{N}|s-t|{ }^{p} d \phi=0\right)$; under the norm $\|s\|_{p}=\left(\int_{N}|s|{ }^{p} d \phi\right)^{1 / p}$, $\mathcal{L}^{p}(\phi)$ is not complete. To obtain a Banach space we let $L^{p}(A)$ consist of Cauchy sequences $s^{(j)}$ of elements of $\mathfrak{L}^{p}(\phi)$ such that $\phi$ transform of each $s^{(j)}$ is bounded and $\lim _{m, n \rightarrow \infty} s^{(m)}-s^{(n)}=0$-we identify two elements $\left\{s^{(j)}\right\}$ and $\left\{t^{(j)}\right\}$ of $L^{p}(\phi)$ if $\lim _{j \rightarrow \infty} \int_{N}\left|s^{(j)}-t^{(j)}\right| p d \phi=0$, where $s^{(j)}, t^{(j)}$ are elements of $\mathfrak{L}^{p}(\phi)$ for each $j$. With the usual definition of addition, scalar multiplication, and norm of equivalence classes of Cauchy sequences we have

For $p>1$ the space $L^{p}(\phi)$ is a Banach space. For $p>1$ the dual space of $L^{p}(\phi)$ is $L^{p^{\prime}}(\phi)$ where $1 / p+1 / p^{\prime}=1$. For each linear continuous functional $F$ on $L^{p}(\phi)$ we have

$$
\begin{aligned}
& F(s)=\int_{N} s t d \phi, \quad t \in L^{p^{\prime}}(\phi), \\
& \|F\|=\left(\int_{N}\|t\| p^{\prime} d \phi\right)^{1 / p^{\prime}}
\end{aligned}
$$

Henceforth we shall not distinguish between an element $a$ and the sequence $\{a, a, \cdots\}$ in $L^{p}(\phi)$.

By $m_{0}$ we denote the Banach space of bounded sequences $s$ with norm given by $\|s\|=\lim \sup \left|s_{n}\right|$ (we identify two sequences $s$ and $t$ if $s-t$ is a null sequence. The dual of $m_{0}$ is $L^{1}(\phi)$, where $\phi$ is a summation method which is regular on null sequences.

For a linear operator $T$ on a space $L^{p}(A)$ where $A$ is a summation matrix to be well defined we must have $\int_{N}|T s|{ }^{p} d A=0$ for all $s$ in $L^{p}(A)$ such that $\int_{N}|s|{ }^{p} d A=0$. On the other hand

If $A$ satisfies (1) and $T$ evaluates to zero each sequence s such that $\int_{N}|s|{ }^{p} d A=0$ then $T$ is well defined.

If $A$ satisfies (1) and

(3) $T$ is representable by a matrix $\left(t_{n k}\right)$ which evaluates to zero each sequence $s$ such that $\int_{N}|s|^{p} d A=0$, then $T$ is well defined. Moreover (3) implies

$$
\operatorname{lub} \sum_{k=0}^{\infty}\left|t_{n k}\right| \rightarrow \infty
$$


so that $T$ transforms each space $L^{p}(A), p>1$, into $m_{0}$. In this case $T$ satisfies

$$
\|T\|^{p^{\prime}} \leqq \lim \sup \sum_{k=0}\left|t_{n k}\right|^{p^{\prime}}
$$

Henceforth $T$ will be assumed to satisfy (3). If $T$ evaluates to zero a sequence $s$ in $L^{p}(A)\left(m_{0}\right)$ such that $\int_{N}|s|{ }^{p} d A>0\left(\lim \sup \left|s_{n}\right|>0\right)$ then zero is an eigenvalue of $T$. If $T=\left(t_{n k}\right)$ is a regular summation, then $T$ (considered as an operator on $L^{p}(A)$ or $m_{0}$ ) has no continuous spectrum. For suppose that zero lies in the continuous spectrum of $T$ (considered an operator on $L^{p}(A)$ ). For each $\epsilon>0$ there is a sequence $s$ such that $\|s\|_{p}=1$ and $\left|\sum t_{n k} s_{k}\right| \leqq \epsilon$ when $n=n_{j}$ where $\left\{n_{j}\right\}$ is a sequence containing $\gamma_{0}$ in its closure. We may adopt Darevsky's technique [4] to construct a sequence $u$, not in $L^{p}(A)$, such that $\left|\sum t_{n k} u_{k}\right| \leqq \epsilon$ when $n=n_{j}$. But this means that $T$ does not have a well defined inverse; zero cannot lie in the continuous spectrum. The proof when $T$ is considered an operator on $m_{0}$ is even simpler.

We note that $\lim _{n \rightarrow \infty} \sum t_{n k}$ is an eigenvalue of $T$ (whenever this limit exists).

Theorem. Suppose that the operator $T=\left(t_{n k}\right)$ satisfies (3) and $\left(4^{\prime}\right)$ and there is a set $E=\left\{n_{j}\right\} \subset N$ such that

$$
\lim _{n \rightarrow \infty ; n \in E} \sum_{K \in E} t_{n, k}=\alpha, \quad \lim _{n \rightarrow \infty ; n \notin E} \sum_{K \in E} t_{n, k}=0,
$$

then $\alpha$ is an eigenvalue of $T$.

Theorem. Let $T_{n}(z)=\sum_{k=0}^{n} t_{n k} z^{k} / z^{n}$. For each number $\alpha$ in $[0,2 \pi]$ such that $\lim _{n \rightarrow \infty} T_{n}\left(e^{i \alpha}\right)$ exists, this limit is an eigenvalue of $T$.

If $B=\left(b_{n, k}\right)$ is a normal regular summation matrix such that

$$
\lim \inf \left|b_{n, n}\right|-\sum_{k=0}^{n-1}\left|b_{n, k}\right|>0
$$

then $B$ has a reciprocal $B^{-1}=\left(\beta_{n, k}\right)$ such that $\operatorname{lub}\left\{\sum_{k=0}^{n}\left|\beta_{n, k}\right|\right\}<\infty$. Hence

If the operator $T$ is representable by a normal summation matrix satisfying (4), $\left(4^{\prime}\right)$ the spectrum of $T$ is contained in the set

$$
\left\{\lambda|\lim \inf | \lambda-t_{n n}\left|-\sum_{k=0}^{n-1}\right| t_{n k} \mid \leqq 0\right\} .
$$

2. Fourier transforms. For each $r, 0<r<1$, let $\mu(r, \theta)$ be a measure on $[0,2 \pi]$ such that 


$$
d \mu(r, \theta)=\sum_{n=0}^{\infty} \hat{\mu}(n) r^{n} e^{i n \theta} d \theta
$$

so that $\mu(r, \theta)$ is analytic with respect to Lebesgue measure for each $r$ in $(0,1)$. The Fourier transform $s$ of a sequence $s$ is defined as a linear functional on a space of measures $\mu(r, \theta)$, and is given by

$$
s(\mu)=\int_{N} s_{n} \hat{\mu}(n) d \propto,
$$

where $a$ is the Abel summation method, whenever the integral on the right-hand side of (5) exists. If we define the $M_{p}$ norm of a measure $\mu$ by

$$
\|\mu\|_{M^{p^{\prime}}}=\operatorname{lub}_{0 \leqq r \leqq 1}\left\{\int\left|\frac{\partial \mu\left(r^{1 / p^{\prime}}, \theta\right)}{\partial \theta}\right|^{p^{\prime}} d \theta\right\}^{1 / p^{\prime}},
$$

then we have

THEOREM. If $p \geqq 2$ then each sequence $s \in \mathscr{L}^{p}(Q)$ has a Fourier transform $\hat{s}(\mu)$ defined for $\mu \in M^{p^{\prime}},\|s\|=\|s\|_{p}$, where $\|s\|$ denotes the norm of $s$ considered as a functional. If for any $p$, the sequence $s \in \mathscr{L}^{p}(\mathbb{Q})$ has a Fourier transform sis such that

$$
\hat{s}(\mu)=0 \text { for all } \mu \text { in } M_{p^{\prime}},
$$

then $s=0$.

THEOREM. If the sequence $\left\{s_{n}\right\}$ has the Fourier transform $s(\mu)$, then for each fixed integer $a$, the sequence $\left\{s_{n+a}\right\}$ has the Fourier transform $e^{i a} s(\mu)$. To each translation-invariant space of sequences $V$, corresponds $a$ subset $E$ of $[0,2 \pi]$ such that $s(\mu)=0$ when $s \in V$ and the measure $\mu(r, \theta)$ is concentrated on $E$.

However, this correspondence is not 1-1. However, if for all increasing subsequence of natural numbers $\left\{n_{k}\right\}$ which tend to infinity we have $\int_{N}\left\{s_{n_{k}} \exp i n_{k}\right\} d Q=0$ then $\int_{N}|s| d Q=0$. Hence

THEOREM. The space of Fourier transforms of sequence $s \in L^{1}(Q)$ may be represented as functions defined on sequences $\left\{\theta_{k}\right\}_{k=1}^{\infty}, 0 \leqq \theta_{k}<2 \pi$. The sequence $s$ is represented by the function $\bar{s}$ :

$$
\tilde{s}\left(\theta_{k}\right)=\sum s_{n_{k}} \exp \left(i n_{k}\right)=s(\mu),
$$

where

$$
n_{k} \equiv \theta_{k}(\bmod 2 \pi), \quad d \mu=\sum_{k=0}^{\infty} \exp \left[i\left(n_{k}+\theta\right)\right] d \theta
$$


So that the Fourier transforms of sequences $s \in \mathcal{L}^{\prime}(Q)$ are the continuous functions on the space $\Theta$ of sequences $\left\{\theta_{\kappa}\right\}$, we topologize $\Theta$ by the metric

$$
d\left(\theta_{k}^{(1)}, \theta_{k}^{(2)}\right)=\int_{D} d a,
$$

where $D=\left\{n_{k} \mid n_{k}^{(1)} \neq n_{k}^{(2)}\right\}$,

$$
n_{k}^{(i)} \equiv \theta_{k}^{(i)}(\bmod 2 \pi), \quad i=1,2
$$

(note that two sequences $\left\{\theta_{k}^{(i)}\right\}$ such that the corresponding $n_{k}^{(i)}$ agree almost everywhere (relative to $Q$ measure) must be identified). The interval $[0,2 \pi]$ with the discrete topology can be embedded in $\Theta$.

Multipliers. A function $f(\theta)$ on $[0,2 \pi]$ is called a multiplier of the space $L^{1}$ if whenever $\hat{s}(\mu)$ is the Fourier transform of a sequence $s \in \mathcal{S}$ then the functional $\hat{s}(f d \mu)$ is the Fourier transform of some sequence $t \in L^{1}$ (the symbol $f d \mu$ denotes the measure with $f$ as its derivative).

THEOREM. The multipliers of $m_{0}$ are the functions $f(\theta)$ such that

$$
f(\theta)=\sum_{n=0}^{\infty} a_{n} e^{i n \theta}, \quad \sum\left|a_{n}\right| \rightarrow \infty ;
$$

for each $p>1$ the multipliers of $L^{p}(A)$ are the trigonometric polynomials.

We conclude with some remarks on sequences $s$ which can be represented by Fourier series

$$
s_{k}=\sum_{n=0}^{\infty} c_{n} \exp \left(i \alpha_{n} k\right), \quad k=0,1, \cdots ;
$$

such sequences are the almost periodic functions on $N$. In [1] I proposed the problem:

Given a sequence of exponents $\left\{\alpha_{n}\right\}$ dense in an interval of length $\pi / 2$, does there exist, for each given subset $E$ of $N$, a series of the form (6) which diverges on $E$ and converges on $N-E$. By a skillful use of Fejer polynomials D. R. Lick has obtained an affirmative answerunfortunately the sequence is not bounded in general. In case the exponents $\alpha_{n}$ are contained in an interval of length $\epsilon<\pi / 2$, then if the series (6) diverges for $k=k_{0}$ it diverges for $\left|k-k_{0}\right| \leqq[\pi / 2 \epsilon]$. If the set of exponents $\left\{\alpha_{n}\right\}$ has only finitely many limit points, then the series (6) converges or diverges for all $k$ according as the series $\sum\left|c_{n}\right|$ converges or diverges. 


\title{
REFERENCES
}

1. G. Brauer, Research Problem: Sets of convergence of exponential series, Bull. Amer. Math. Soc. 72 (1966), p. 470.

2. L. Gillman and M. Jerison, Rings of continuous functions, Van Nostrand, New York, 1960. 1958.

3. N. Dunford and J. T. Schwartz, Linear operators. I, Interscience, New York,

4. V. M. Darevsky, On intrinsically perfect methods of summation, Bull. Acad. Sci. URSS Ser. Mat. (Izv. Akad. Nauk SSSR Ser. Mat.) 10 (1946), 97-104.

UNIVERSity OF MinNesota

\section{THE UNION OF FLAT $(n-1)$-BALLS IS FLAT IN $R^{n}$}

\author{
BY ROBION C. KIRBY ${ }^{1}$
}

Communicated by Eldon Dyer, December 15, 1967

TheOREM. ${ }^{2}$ Let $\beta_{1}^{n-1}$ and $\beta_{2}^{n-1}$ be two locally flat $(n-1)$-balls in $R^{n}$ with $\beta_{1} \cap \beta_{2}=\partial \beta_{1} \cap \partial \beta_{2}=\beta^{n-2}$, where $\beta^{n-2}$ is an $(n-2)$-ball which is locally flat in $\partial \beta_{1}$ and $\partial \beta_{2}$. Then $\beta_{1} \cup \beta_{2}$ is a flat $(n-1)$-ball in $R^{n}$.

This result has been announced by Cernavskiǐ [1], but only for $n \geqq 5$ since his outlined proof uses engulfing. Our proof avoids engulfing and works for all $n$; a thorough knowledge of Cantrell and Lacher's version (see $[2, \S \S 4$ and 5]) of Cernavski1's theorem is necessary to understand our proof.

We also have another proof of the following corollary which appears in [4].

CoROLLARY. Let $g: M^{n-1} \rightarrow N^{n}$ be an imbedding of an (n-1)-manifold into an n-manifold which is locally flat except on a set $E$. If $n>3$, then $E$ contains no isolated points (see [3] for the same result when $M$ and $N$ are spheres).

Proof. Let $C$ be a neighborhood of an isolated point $p$ in $M$ which is homeomorphic to an $(n-1)$-ball, with $g$ locally flat on $C-p$. Then split $C$ into $(n-1)$-balls $C_{1}$ and $C_{2}$ so that $C=C_{1} \cup C_{2}$ and $C_{1} \cap C_{2}$ is an $(n-2)$-ball containing $p . g$ is locally flat on $C_{1}$ and $C_{2}$ except at the point $p$ on their boundaries. Then, since $n>3, g$ is flat on all of $C_{1}$ and $C_{2}$ by [5]. It follows from the theorem that $C_{1} \cup C_{2}=C$ is flat, so $E$ has no isolated points.

1 Research supported by NSF Grant 6530 .

2 Added in proof. Cernavskil has independently proven this theorem by similar methods. 